\title{
THE ENERGY TECHNIQUE FOR THE SIX-STEP BDF METHOD
}

\author{
GEORGIOS AKRIVIS, MINGHUA CHEN, FAN YU, AND ZHI ZHOU
}

\begin{abstract}
In combination with the Grenander-Szegö theorem, we observe that a relaxed positivity condition on multipliers, milder than the basic requirement of the NevanlinnaOdeh multipliers that the sum of the absolute values of their components is strictly less than 1, makes the energy technique applicable to the stability analysis of BDF methods for parabolic equations with selfadjoint elliptic part. This is particularly useful for the six-step BDF method for which no Nevanlinna-Odeh multiplier exists. We introduce multipliers satisfying the positivity property for the six-step BDF method and establish stability of the method for parabolic equations.
\end{abstract}

\section{INTRODUCTION}

Let $T>0, u^{0} \in H$, and consider the initial value problem of seeking $u \in C((0, T] ; D(A)) \cap$ $C([0, T] ; H)$ satisfying

$$
\left\{\begin{array}{l}
u^{\prime}(t)+A u(t)=0, \quad 0<t<T \\
u(0)=u^{0}
\end{array}\right.
$$

with $A$ a positive definite, selfadjoint, linear operator on a Hilbert space $(H,(\cdot, \cdot))$ with domain $D(A)$ dense in $H$.

We consider the $q$-step backward difference formula (BDF) method, generated by the polynomials $\alpha$ and $\beta$,

$$
\alpha(\zeta)=\sum_{j=1}^{q} \frac{1}{j} \zeta^{q-j}(\zeta-1)^{j}=\sum_{j=0}^{q} \alpha_{j} \zeta^{j}, \quad \beta(\zeta)=\zeta^{q} .
$$

The BDF methods are $A\left(\vartheta_{q}\right)$-stable with $\vartheta_{1}=\vartheta_{2}=90^{\circ}, \vartheta_{3} \approx 86.03^{\circ}, \vartheta_{4} \approx 73.35^{\circ}, \vartheta_{5} \approx$ $51.84^{\circ}$ and $\vartheta_{6} \approx 17.84^{\circ}$; see [9, Section V.2]. Exact values of $\vartheta_{q}, q=3,4,5,6$, are given in [4]. The order of the $q$-step method is $q$.

Let $N \in \mathbb{N}, \tau:=T / N$ be the time step, and $t^{n}:=n \tau, n=0, \ldots, N$, be a uniform partition of the interval $[0, T]$. We recursively define a sequence of approximations $u^{m}$ to the nodal values $u\left(t^{m}\right)$ by the $q$-step BDF method,

$$
\sum_{i=0}^{q} \alpha_{i} u^{n+i}+\tau A u^{n+q}=0, \quad n=0, \ldots, N-q,
$$

assuming that starting approximations $u^{0}, \ldots, u^{q-1}$ are given.

Date: July 20, 2020.

2010 Mathematics Subject Classification. Primary 65M12, 65M60; Secondary 65L06.

Key words and phrases. Six-step BDF method, multipliers, parabolic equations, stability estimate, energy technique.

The research of M. Chen and F. Yu is partially supported by NSFC 11601206, and the research of Z. Zhou is supported by Hong Kong RGC grant (No. 25300818). 
Let $|\cdot|$ denote the norm on $H$ induced by the inner product $(\cdot, \cdot)$, and introduce on $V, V:=D\left(A^{1 / 2}\right)$, the norm $\|\cdot\|$ by $\|v\|:=\left|A^{1 / 2} v\right|$. We identify $H$ with its dual, and denote by $V^{\prime}$ the dual of $V$, and by $\|\cdot\|_{\star}$ the dual norm on $V^{\prime},\|v\|_{\star}=\left|A^{-1 / 2} v\right|$. We shall use the notation $(\cdot, \cdot)$ also for the antiduality pairing between $V^{\prime}$ and $V$.

Stability of the A-stable one- and two-step BDF methods (1.3) can be easily established by the energy method. The powerful Nevanlinna-Odeh multiplier technique extends the applicability of the energy method to the non A-stable three-, four- and five-step BDF methods. In contrast, as we shall see, no Nevanlinna-Odeh multiplier exists for the sixstep BDF method. Here, we show that, in combination with the Grenander-Szegö theorem, the energy technique is applicable even with multipliers satisfying milder requirements than Nevanlinna-Odeh multipliers. We introduce such multipliers for the six-step BDF method and prove stability by the energy technique.

An outline of the paper is as follows: In Section 2, we relax the requirements on the multipliers for BDF methods and present multipliers for the six-step BDF method. In Section 3, we use a new multiplier in combination with the Grenander-Szegö theorem and prove stability of the six-step BDF method for the initial value problem (1.1).

\section{MUltipliers FOR THE SIX-STEP BDF METHOD}

Multipliers for the three-, four- and five-step BDF methods were introduced by Nevanlinna and Odeh already in 1981, see [12], to make the energy method applicable to the stability analysis of these methods for parabolic equations; no multipliers are required for the A-stable one- and two-step BDF methods. The multiplier technique became widely known and popular after its first actual application to the stability analysis for parabolic equations by Lubich, Mansour, and Venkataraman in 2013; see [11].

The multiplier technique hinges on the celebrated equivalence of A- and G-stability for multistep methods by Dahlquist; see [8].

Lemma 2.1 ([8]; see also [6] and [9, Section V.6]). Let $\alpha(\zeta)=\alpha_{q} \zeta^{q}+\cdots+\alpha_{0}$ and $\mu(\zeta)=\mu_{q} \zeta^{q}+\cdots+\mu_{0}$ be polynomials, with real coefficients, of degree at most $q$ (and at least one of them of degree $q)$ that have no common divisor. Let $(\cdot, \cdot)$ be a real inner product with associated norm $|\cdot|$. If

$$
\operatorname{Re} \frac{\alpha(\zeta)}{\mu(\zeta)}>0 \quad \text { for }|\zeta|>1
$$

then there exists a positive definite symmetric matrix $G=\left(g_{i j}\right) \in \mathbb{R}^{q, q}$ and real $\delta_{0}, \ldots, \delta_{q}$ such that for $v^{0}, \ldots, v^{q}$ in the inner product space,

$$
\left(\sum_{i=0}^{q} \alpha_{i} v^{i}, \sum_{j=0}^{q} \mu_{j} v^{j}\right)=\sum_{i, j=1}^{q} g_{i j}\left(v^{i}, v^{j}\right)-\sum_{i, j=1}^{q} g_{i j}\left(v^{i-1}, v^{j-1}\right)+\left|\sum_{i=0}^{q} \delta_{i} v^{i}\right|^{2} .
$$

Definition 2.1 (Multipliers and Nevanlinna-Odeh multipliers). Let $\alpha$ be the generating polynomial of the $q$-step BDF method defined in (1.2). Consider a $q$-tuple $\left(\mu_{1}, \ldots, \mu_{q}\right)$ of real numbers such that with the given $\alpha$ and $\mu(\zeta):=\zeta^{q}-\mu_{1} \zeta^{q-1}-\cdots-\mu_{q}$, the pair $(\alpha, \mu)$ satisfies the A-stability condition (A), and, in addition, the polynomials $\alpha$ and $\mu$ have no common divisor. Then, we call $\left(\mu_{1}, \ldots, \mu_{q}\right)$ Nevanlinna-Odeh multiplier for the $q$-step BDF method if

$$
1-\left|\mu_{1}\right|-\cdots-\left|\mu_{q}\right|>0
$$


and simply multiplier if it satisfies the positivity property

$$
1-\mu_{1} \cos x-\cdots-\mu_{q} \cos (q x)>0 \quad \forall x \in \mathbb{R} .
$$

Notice that, with the notation of this definition, $(A)$ and $(G)$, respectively, mean that the $q$-step scheme described by the parameters $\alpha_{q}, \ldots, \alpha_{0}, 1,-\mu_{1}, \ldots,-\mu_{q}$ and the corresponding one-leg method are A- and G-stable, respectively. Of course, these are necessarily low order methods but this is irrelevant here; we do not compute with them; we only use them to establish stability of the $q$-step BDF method.

Optimal Nevanlinna-Odeh multipliers, i.e., the ones with minimal $\left|\mu_{1}\right|+\cdots+\left|\mu_{q}\right|$, for the three-, four- and five-step BDF methods were given in [3].

Some comments on the requirements in Definition 2.1 and their role in the stability analysis are in order. To prove stability of the method by the energy technique, we test (1.3) by $u^{n+q}-\mu_{1} u^{n+q-1}-\cdots-\mu_{q} u^{n}$ and obtain

$$
\left(\sum_{i=0}^{q} \alpha_{i} u^{n+i}, u^{n+q}-\sum_{j=1}^{q} \mu_{j} u^{n+q-j}\right)+\tau\left(A u^{n+q}, u^{n+q}-\sum_{j=1}^{q} \mu_{j} u^{n+q-j}\right)=0
$$

$n=0, \ldots, N-q$. The first term on the left-hand side can be estimated from below using $(\mathrm{G})$; this is the motivation for the requirement $(\mathrm{A})$. Which one of the other two conditions, (P1) or (P2), enters into the stability analysis, depends on the way we handle the second term on the left-hand side of (2.1). If we estimate this term from below at every time level and then sum over $n$, requirement (P1) is crucial; cf., e.g., [5], [1], [3]. Instead, if we sum over $n$ and subsequently estimate the sum of the second terms, the relaxed positivity condition (P2) suffices. In the latter approach, in view of the Grenander-Szegö theorem, (P2) ensures that symmetric band Toeplitz matrices, of any dimension, with generating function the positive trigonometric polynomial $(1-\varepsilon)-\mu_{1} \cos x-\cdots-\mu_{q} \cos (q x)$, for sufficiently small $\varepsilon$, are positive definite; see section 3 .

It is well known that any multiplier for the $q$-step BDF method satisfies the property

$$
\left|\mu_{1}\right|+\cdots+\left|\mu_{q}\right| \geqslant \cos \vartheta_{q}
$$

see [12]. In particular, for the six-step BDF method this means that $\left|\mu_{1}\right|+\cdots+\left|\mu_{6}\right| \geqslant$ 0.9516169. Actually, as we shall see, no Nevanlinna-Odeh multiplier exists for the sixstep BDF method; see Remark 2.1. This was the motivation for our relaxation on the requirements for multipliers. Fortunately, the relaxed positive condition (P2) leads to a positive result.

Proposition 2.1 (A multiplier for the six-step BDF method). The set of numbers

$$
\mu_{1}=\frac{13}{9}, \quad \mu_{2}=-\frac{25}{36}, \quad \mu_{3}=\frac{1}{9}, \quad \mu_{4}=\mu_{5}=\mu_{6}=0
$$

is a multiplier for the six-step BDF method.

Proof. The proof consists of two parts; we first prove the A-stability property (A) and subsequently the positivity property (P2).

A-stability property (A). The corresponding polynomial $\mu$ is

$$
\mu(\zeta)=\zeta^{3}\left(\zeta-\frac{1}{2}\right)^{2}\left(\zeta-\frac{4}{9}\right)=\zeta^{6}-\frac{13}{9} \zeta^{5}+\frac{25}{36} \zeta^{4}-\frac{1}{9} \zeta^{3}=\frac{1}{36} \zeta^{3}\left(36 \zeta^{3}-52 \zeta^{2}+25 \zeta-4\right)
$$

We recall the generating polynomial $\alpha$ of the six-step BDF method,

$$
60 \alpha(\zeta)=147 \zeta^{6}-360 \zeta^{5}+450 \zeta^{4}-400 \zeta^{3}+225 \zeta^{2}-72 \zeta+10 .
$$


First, $\alpha(1 / 2)=-37 / 3840$ and $\alpha(4 / 9)=-0.003730423508913$, whence the polynomials $\alpha$ and $\mu$ have no common divisor.

Now, $\alpha(z) / \mu(z)$ is holomorphic outside the unit disk in the complex plane, and

$$
\lim _{|z| \rightarrow \infty} \frac{\alpha(z)}{\mu(z)}=\alpha_{6}=\frac{147}{60}>0 .
$$

Therefore, according to the maximum principle for harmonic functions, the A-stability property $(\mathrm{A})$ is equivalent to

$$
\operatorname{Re} \frac{\alpha(\zeta)}{\mu(\zeta)} \geqslant 0 \quad \forall \zeta \in \mathscr{K}
$$

with $\mathscr{K}$ the unit circle in the complex plane, $\mathscr{K}:=\{\zeta \in \mathbb{C}:|\zeta|=1\}$, i.e., equivalent to

$$
\operatorname{Re}\left[\alpha\left(\mathrm{e}^{\mathrm{i} \varphi}\right) \mu\left(\mathrm{e}^{-\mathrm{i} \varphi}\right)\right] \geqslant 0 \quad \forall \varphi \in \mathbb{R} .
$$

In view of (2.4), the desired property (2.5) takes the form

$$
\operatorname{Re}\left[60 \alpha\left(\mathrm{e}^{\mathrm{i} \varphi}\right) \mathrm{e}^{-\mathrm{i} 3 \varphi}\left(36 \mathrm{e}^{-\mathrm{i} 3 \varphi}-52 \mathrm{e}^{-\mathrm{i} 2 \varphi}+25 \mathrm{e}^{-\mathrm{i} \varphi}-4\right)\right] \geqslant 0 \quad \forall \varphi \in \mathbb{R} .
$$

Now, it is easily seen that

$$
\begin{aligned}
60 \alpha\left(\mathrm{e}^{\mathrm{i} \varphi}\right) \mathrm{e}^{-\mathrm{i} 3 \varphi} & =[157 \cos (3 \varphi)-432 \cos (2 \varphi)+675 \cos \varphi-400] \\
& +\mathrm{i}[137 \sin (3 \varphi)-288 \sin (2 \varphi)+225 \sin \varphi] .
\end{aligned}
$$

With $x:=\cos \varphi$, recalling the elementary trigonometric identities

$$
\cos (2 \varphi)=2 x^{2}-1, \cos (3 \varphi)=4 x^{3}-3 x, \sin (2 \varphi)=2 x \sin \varphi, \sin (3 \varphi)=\left(4 x^{2}-1\right) \sin \varphi,
$$

we easily see that

$$
60 \alpha\left(\mathrm{e}^{\mathrm{i} \varphi}\right) \mathrm{e}^{-\mathrm{i} 3 \varphi}=4(1-x)\left(8+59 x-157 x^{2}\right)+\mathrm{i} 4\left(137 x^{2}-144 x+22\right) \sin \varphi .
$$

Notice that the factor $1-x$ in the real part of $\alpha\left(\mathrm{e}^{\mathrm{i} \varphi}\right) \mathrm{e}^{-\mathrm{i} 3 \varphi}$ is due to the fact that $\alpha(1)=0$. Similarly,

$$
\begin{aligned}
36 \mathrm{e}^{-\mathrm{i} 3 \varphi}-52 \mathrm{e}^{-\mathrm{i} 2 \varphi}+25 \mathrm{e}^{-\mathrm{i} \varphi}-4 & =[36 \cos (3 \varphi)-52 \cos (2 \varphi)+25 \cos \varphi-4] \\
& -\mathrm{i}[36 \sin (3 \varphi)-52 \sin (2 \varphi)+25 \sin \varphi]
\end{aligned}
$$

and

$$
\begin{aligned}
36 \mathrm{e}^{-\mathrm{i} 3 \varphi}-52 \mathrm{e}^{-\mathrm{i} 2 \varphi}+25 \mathrm{e}^{-\mathrm{i} \varphi}-4 & =\left(144 x^{3}-104 x^{2}-83 x+48\right) \\
& -\mathrm{i}\left(144 x^{2}-104 x-11\right) \sin \varphi .
\end{aligned}
$$

In view of (2.7) and (2.8), the desired property (2.6) can be written in the form

$$
4(1-x) P(x) \geqslant 0 \quad \forall x \in[-1,1]
$$

with

$$
\begin{aligned}
P(x) & :=\left(8+59 x-157 x^{2}\right)\left(144 x^{3}-104 x^{2}-83 x+48\right) \\
& +(1+x)\left(137 x^{2}-144 x+22\right)\left(144 x^{2}-104 x-11\right),
\end{aligned}
$$

i.e.,

$$
P(x)=2\left(71+611 x+1334 x^{2}-5150 x^{3}+4784 x^{4}-1440 x^{5}\right) .
$$

It is now easy to see that $P$ is positive in the interval $[-1,1]$, and thus that $(2.6)$ is valid. First, the quadratic polynomial $71+611 x+1334 x^{2}$ is positive for all real $x$, since it does not have real roots. All other terms are positive for negative $x$, whence $P(x)$ is positive 
for negative $x$. Furthermore, for $0 \leqslant x \leqslant 1$, we obviously have $71+611 x \geqslant 682 x^{2}$, and can estimate $P(x)$ from below as follows

$$
\begin{aligned}
P(x) & \geqslant 2 x^{2}\left(2016-5150 x+4784 x^{2}-1440 x^{3}\right) \\
& =2 x^{2}\left[\left(2016-5150 x+3344 x^{2}\right)+1440 x^{2}(1-x)\right] .
\end{aligned}
$$

Again, the quadratic polynomial $2016-5150 x+3344 x^{2}$ is positive for all real $x$, and the positivity of $P(x)$ follows.

Positivity property (P2). To prove the desired positivity property (P2) for the multiplier (2.3), we consider the function $f$,

$$
f(x):=\frac{31}{32}-\frac{13}{9} \cos x+\frac{25}{36} \cos (2 x)-\frac{1}{9} \cos (3 x), \quad x \in \mathbb{R} .
$$

Now, elementary trigonometric identities lead to the following form of $f$

$$
f(x)=-\frac{4}{9} \cos ^{3} x+\frac{25}{18} \cos ^{2} x-\frac{10}{9} \cos x+\frac{79}{288} .
$$

Hence, we consider the polynomial $p$,

$$
p(x):=-\frac{4}{9} x^{3}+\frac{25}{18} x^{2}-\frac{10}{9} x+\frac{79}{288}, \quad x \in[-1,1] .
$$

It is easily seen that $p$ attains its minimum at $x^{\star}=(25-\sqrt{145}) / 24$ and

$$
p\left(x^{\star}\right)=0.009321552602567>0 .
$$

Therefore, $f$ is indeed positive; in particular, the desired positivity property (P2) is satisfied. See also Figure 2.1.
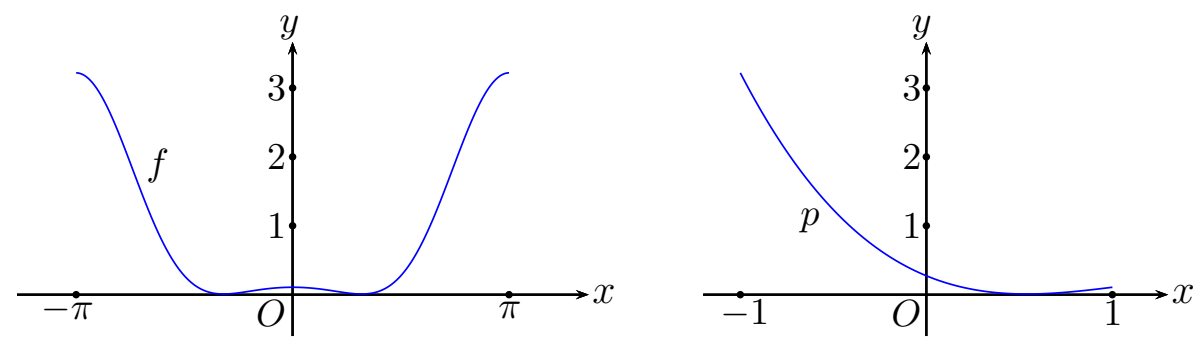

FiguRE 2.1. The graphs of the function $f$ and the polynomial $p$ of (2.11) and (2.12).

2.1. On the conditions (P2) and (P1). We briefly comment on the discrepancy between the conditions (P2) and (P1). Obviously, (P1) implies (P2).

Let $S_{q} \subset \mathbb{R}^{q}$ denote the region of the points $\left(\mu_{1}, \ldots, \mu_{q}\right)$ satisfying the positivity condition (P2). Since (P1) and (P2) are obviously equivalent for $q$-tuples $\left(\mu_{1}, \ldots, \mu_{q}\right)$ with only one nonvanishing component, the intersection of $S_{q}$ with each coordinate axis is an interval of the form $(-1,1)$.

Let us next focus on the instrumental case of the intersection of $S_{q}$ with the $\mu_{1} \mu_{2}$ plane, i.e., consider the set of points $\left(\mu_{1}, \ldots, \mu_{q}\right) \in S_{q}$ with $\mu_{3}=\cdots=\mu_{q}=0$. Then, the positivity condition reads

$$
p(x):=1-\mu_{1} x-\mu_{2}\left(2 x^{2}-1\right)>0, \quad x \in[-1,1] .
$$


For $\mu_{2}=0$, this condition is satisfied if and only if $\left|\mu_{1}\right|<1$. For nonvanishing $\mu_{2}$, the derivative of $p$ vanishes at $x^{\star}=-\mu_{1} /\left(4 \mu_{2}\right)$ and

$$
p\left(x^{\star}\right)=1+\mu_{2}+\frac{1}{8} \frac{\mu_{1}^{2}}{\mu_{2}} .
$$

For positive $\mu_{2}$, this is a positive global maximum of $p$. Therefore, in this case (2.13) is satisfied if and only if $p(-1)$ and $p(1)$ are positive, whence

$$
\mu_{2}<1-\left|\mu_{1}\right| \text {. }
$$

For negative $\mu_{2}$, the expression in (2.14) is a global minimum of $p$. Now, we distinguish two subcases. It $\left|\mu_{2}\right| \leqslant\left|\mu_{1}\right| / 4$, then the minimum is attained at a point $\left|x^{\star}\right| \geqslant 1$, whence (2.15) suffices for (2.13). If, on the other hand, $\left|x^{\star}\right|<1$, then (2.13) is satisfied if and only if the expression on the right-hand side of (2.14) is positive, i.e.,

$$
4\left(\mu_{2}+\frac{1}{2}\right)^{2}+\frac{1}{2} \mu_{1}^{2}<1
$$

that is, $\left(\mu_{1}, \mu_{2}\right)$ lies in the interior of an ellipse. Summarizing, (2.13) is satisfied if and only if $\left(\mu_{1}, \mu_{2}\right)$ lie in the region

$S:=\left\{\left(\mu_{1}, \mu_{2}\right):-\frac{\left|\mu_{1}\right|}{4} \leqslant \mu_{2}<1-\left|\mu_{1}\right|\right\} \cup\left\{\left(\mu_{1}, \mu_{2}\right): 4\left(\mu_{2}+\frac{1}{2}\right)^{2}+\frac{1}{2} \mu_{1}^{2}<1\right.$ and $\left.\left|\mu_{2}\right|>\frac{\left|\mu_{1}\right|}{4}\right\}$.

Notice that the lines $\mu_{2}= \pm\left(1-\mu_{1}\right)$ are tangent to the ellipse at their intersection points with the lines $\mu_{2}=\mp \mu_{1} / 4$, respectively, i.e., at the points $( \pm 4 / 3,-1 / 3)$. This is, of course, due to the fact that for these values the global minimum in (2.14) is attained at the points $x^{\star}= \pm 1$. Therefore, the intersection $S$ of $S_{q}$ with the $\mu_{1} \mu_{2}$ plane is the union of two overlapping simple sets, a triangle and an ellipse,

$$
S=\left\{\left(\mu_{1}, \mu_{2}\right):-\frac{1}{3} \leqslant \mu_{2}<1-\left|\mu_{1}\right|\right\} \cup\left\{\left(\mu_{1}, \mu_{2}\right): 4\left(\mu_{2}+\frac{1}{2}\right)^{2}+\frac{1}{2} \mu_{1}^{2}<1\right\} ;
$$

see Figure 2.2, right. Notice, in particular, that

$$
\left|\mu_{1}\right|<\sqrt{2} \text { and }\left|\mu_{2}\right|<1 \text {. }
$$

Replacing $x$ by $x / 2$ and by $x / 3$, respectively, in the positivity condition (P2), it is obvious that the intersection of $S_{q}$ with the $\mu_{2} \mu_{4}$ plane, for $q \geqslant 4$, and with the $\mu_{3} \mu_{6}$ plane, for $q=6$, respectively, is of the form $(2.16)$ with $\left(\mu_{1}, \mu_{2}\right)$ replaced by $\left(\mu_{2}, \mu_{4}\right)$ and by $\left(\mu_{3}, \mu_{6}\right)$, respectively.

2.2. On the construction of multipliers. In this part, we describe some necessary conditions of multipliers satisfying the A-stability condition (A) and the relaxed positive condition (P2). To begin with, we show that no multiplier with $\mu_{3}=\cdots=\mu_{6}=0$ exists.

Proposition 2.2. There is no multiplier with $\mu_{3}=\cdots=\mu_{6}=0$, satisfying (A) and (P2).

Proof. The positivity condition (P2) is satisfied if and only if

$$
1-\mu_{1} x-\mu_{2}\left(2 x^{2}-1\right)>0 \quad \forall x \in[-1,1]
$$

see (2.13). The A-stability condition (A) is in this case equivalent to (2.9) with

$$
\begin{aligned}
P(x)= & \left(8+59 x-157 x^{2}\right)\left(4 x^{3}-\mu_{1}\left(2 x^{2}-1\right)-3 x-\mu_{2} x\right) \\
& +(1+x)\left(137 x^{2}-144 x+22\right)\left(4 x^{2}-2 \mu_{1} x-\mu_{2}-1\right) .
\end{aligned}
$$



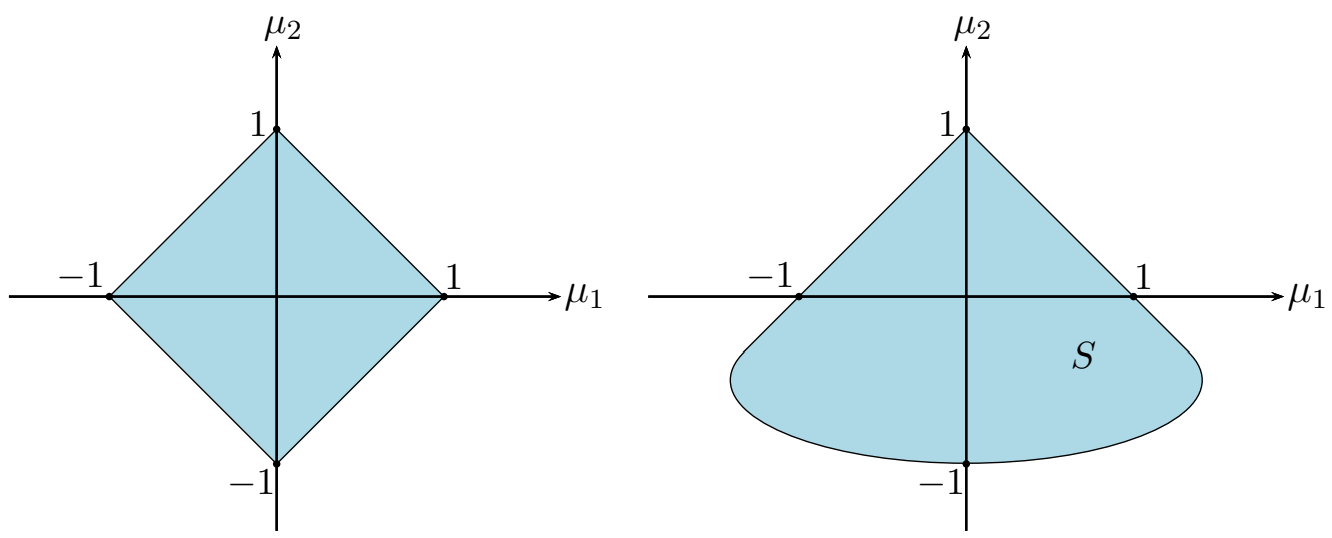

Figure 2.2. Illustration of the conditions (P1) and (P2), left and right, respectively, for $\mu_{3}=\cdots=\mu_{6}=0$; cf. (2.16).

First, the estimate $\left|\mu_{1}\right|<\sqrt{2}$ in (2.17) and the nonnegativity of

$$
P(-4 / 25)=-41.65312 \mu_{2}+7.86979 \mu_{1}-39.13478
$$

lead to

$$
\mu_{2}<\frac{7.86979 \sqrt{2}-39.13478}{41.65312}=-0.672343782385853 .
$$

On the other hand, for $\mu_{2}<-0.672343782385853$, we have $\left|\mu_{2}\right|>\left|\mu_{1}\right| / 4$, and thus $\left(\mu_{1}, \mu_{2}\right)$ must lie in the interior of the ellipse in (2.16). Now, $P(0.99)=a \mu_{2}+b \mu_{1}+c$ with

$$
a=\frac{2086460708677967}{35184372088832}, \quad b=\frac{1053766469372221}{35184372088832}, \quad c=\frac{9685378027}{109951162777600},
$$

and the intersection points of the line $P(0.99)=0$ and the ellipse $4\left(\mu_{2}+1 / 2\right)^{2}+\mu_{1}^{2} / 2=1$ are

$$
\left\{\begin{array}{l}
A=\left(2.941186035762484 \cdot 10^{-6},-1.08131109678632 \cdot 10^{-12}\right), \\
B=(1.328818676149621,-0.671118740185537) .
\end{array}\right.
$$

It is easily seen that $P(0.99)$ is nonnegative only in the part of the interior of the ellipse to the right of the segment $A B$; cf. Figure 2.3. Therefore, $P(0.99) \geqslant 0$ implies

$$
\mu_{2} \geqslant-0.671118740185537 \text {. }
$$

This together with (2.2) leads to a contradiction; hence, no multiplier of the form $\left(\mu_{1}, \mu_{2}, 0\right.$, $\ldots, 0)$ exists for the six-step BDF method.

Our next attempt was to seek a multiplier with $\mu_{4}=\mu_{5}=\mu_{6}=0$. In this case, the A-stability condition (A) and the positivity condition (P2) lead, respectively, to the conditions

$$
\begin{aligned}
P(x)= & \left(8+59 x-157 x^{2}\right)\left(4 x^{3}-\mu_{1}\left(2 x^{2}-1\right)-3 x-\mu_{2} x-\mu_{3}\right) \\
& +(1+x)\left(137 x^{2}-144 x+22\right)\left(4 x^{2}-2 \mu_{1} x-\mu_{2}-1\right) \geqslant 0 \quad \forall x \in[-1,1]
\end{aligned}
$$

and

$$
f(x):=1-\mu_{1} \cos x-\mu_{2} \cos (2 x)-\mu_{3} \cos (3 x)>0 \quad \forall x \in \mathbb{R} .
$$




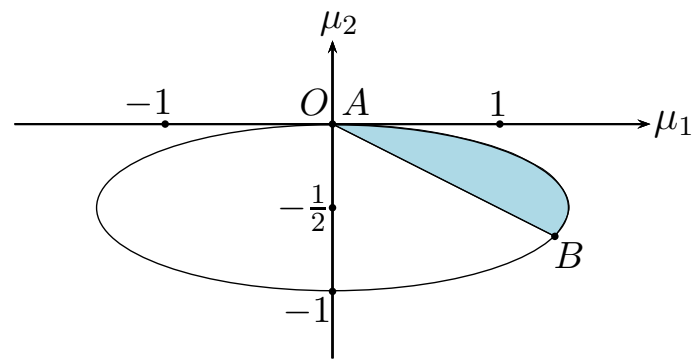

Figure 2.3. Out of the interior points $\left(\mu_{1}, \mu_{2}\right)$ of the ellipse, $P(0.99)$, see (2.19), is nonnegative only in the blue region; in the blue region, $\mu_{2} \geqslant-0.671118740185535$. The points $A$ and $B$ are given in (2.21). The discrepancy between $A$ and $O=(0,0)$ is invisible.

Necessary conditions for (2.22) and (2.23) could be derived by evaluating $P$ and $f$ at certain points. For instance, we claim the following necessary condition, which helps us to construct multipliers.

Proposition 2.3. If $\left(\mu_{1}, \mu_{2}, \mu_{3}, 0,0,0\right)$ is a multiplier of the six-step BDF method, then there holds

$$
0.41990729<\mu_{1}<\sqrt{3},-1<\mu_{2}<-0.58852878,0<\mu_{3}<1,\left|\mu_{1}\right|+\left|\mu_{2}\right|+\left|\mu_{3}\right|>1 .
$$

Proof. First, $\left|\mu_{2}\right|<1$ follows immediately from the positivity of $f(\pi / 2)$ and of $f(0)$ and $f(\pi)$. Furthermore,

$$
2 f(2 \pi / 3)+f(0)=3\left(1-\mu_{3}\right) \text { and } 2 f(\pi / 3)+f(\pi)=3\left(1+\mu_{3}\right),
$$

whence $\left|\mu_{3}\right|<1$. In view of

$$
f(\pi / 6)=\frac{1}{2}\left(-\sqrt{3} \mu_{1}-\mu_{2}+2\right) \quad \text { and } \quad f(5 \pi / 6)=\frac{1}{2}\left(\sqrt{3} \mu_{1}-\mu_{2}+2\right),
$$

we have $\sqrt{3}\left|\mu_{1}\right|<2-\mu_{2}$, and, in combination with $\mu_{2}>-1$, infer that $\left|\mu_{1}\right|<\sqrt{3}$.

Up to this point, we did not use the nonnegativity of $P$. Now we check $P(0) \geqslant 0$, i.e.,

$$
P(0)=2\left[4\left(\mu_{1}-\mu_{3}\right)-11\left(1+\mu_{2}\right)\right] \geqslant 0 .
$$

Since $1+\mu_{2}>0$, we infer that $\mu_{3}<\mu_{1}$. Furthermore, since $\mu_{1}<\sqrt{3}$ and $\left|\mu_{3}\right|<1$,

$$
11 \mu_{2}<4(\sqrt{3}+1)-11<-0.07179 \text {, whence } \mu_{2}<-0.65263636 \cdot 10^{-2} \text {. }
$$

Meanwhile, since $274 / 625+1154 \mu_{2} / 25<0$, the nonegativity of

$$
P(0.8)=\frac{274}{625}+\frac{1154}{25} \mu_{2}+\frac{3572}{125} \mu_{1}+\frac{1132}{25} \mu_{3}
$$

yields $3572 \mu_{1} / 125+1132 \mu_{3} / 25>0$, which together with $\mu_{3}<\mu_{1}$ leads to

$$
\frac{3572}{125} \mu_{1}+\frac{1132}{25} \mu_{1}>\frac{3572}{125} \mu_{1}+\frac{1132}{25} \mu_{3}>0,
$$

i.e., $\mu_{1}>0$. Therefore, we arrive at

$$
0<\mu_{1}<\sqrt{3}, \quad-1<\mu_{2}<-0.65263636 \cdot 10^{-2} \text { and } 0<\left|\mu_{3}\right|<1 \text {. }
$$

Next, we prove $\mu_{3}>0$ by contradiction. If $\mu_{3} \leqslant 0$, then the positivity of $f(\pi / 4)$ yields

$$
f(\pi / 4)=1-\frac{\sqrt{2}}{2}\left(\mu_{1}-\mu_{3}\right)>0 \Longrightarrow \mu_{1}<\sqrt{2} .
$$


This and the nonnegativity of $P(-4 / 25)$ imply $\mu_{2}<-0.672$. Then, we can derive a lower bound $\mu_{1}>1.3426$ by examining $P(0.999) \geqslant 0$. However, with $\mu_{1}>1.3426, \mu_{2}<-0.672$ and $\mu_{3} \leqslant 0$, it is easy to observe that

$$
2 f(\pi / 3)=-\mu_{1}+\mu_{2}+2 \mu_{3}+2 \leqslant-1.3426-0.672+2<-0.0146,
$$

which violates the positive condition (2.23). Therefore, we conclude that $\mu_{3}>0$.

Moreover, from $\mu_{1}<\sqrt{3}, \mu_{3}>0$ and the nonnegativity of

$$
P(-66 / 625)=7.33518936 \mu_{1}-34.64182239 \mu_{2}-0.01883648 \mu_{3}-33.09263039,
$$

we infer that

$$
\mu_{2}<\frac{7.33518936 \sqrt{3}-33.09263039}{34.64182239}<-0.58852878
$$

Then, the nonnegativity of $P(27 / 125)$ yields $\mu_{1}>0.41990729$. Thus, we arrive at

$$
0.41990729<\mu_{1}<\sqrt{3}, \quad-1<\mu_{2}<-0.58852878 \text { and } 0<\mu_{3}<1 .
$$

Finally, the property $\left|\mu_{1}\right|+\left|\mu_{2}\right|+\left|\mu_{3}\right|>1$ is a special case of the more general result of the next Remark.

Remark 2.1 (Nonexistence of Nevanlinna-Odeh multipliers for the six-step BDF method). The multiplier (2.3) is not unique. In general, the A-stability condition (A) and the positivity condition (P2) lead to the conditions

$$
\begin{aligned}
P(x)= & \left(-80 x^{5}+208 x^{4}-122 x^{3}-82 x^{2}+98 x-22\right)+\left(40 x^{4}-104 x^{3}+71 x^{2}+15 x+8\right) \mu_{1} \\
& +\left(20 x^{3}-52 x^{2}+114 x-22\right) \mu_{2}-\left(8+59 x-157 x^{2}\right) \mu_{3} \\
& +\left(294 x^{3}-66 x^{2}-130 x+22\right) \mu_{4}+\left(588 x^{4}-132 x^{3}-417 x^{2}+103 x+8\right) \mu_{5} \\
& +\left(1176 x^{5}-264 x^{4}-1128 x^{3}+272 x^{2}+146 x-22\right) \mu_{6} \geqslant 0
\end{aligned}
$$

and

$$
\begin{aligned}
p(x)= & 1-x \mu_{1}-\left(2 x^{2}-1\right) \mu_{2}-\left(4 x^{3}-3 x\right) \mu_{3}-\left(8 x^{4}-8 x^{2}+1\right) \mu_{4} \\
& -\left(16 x^{5}-20 x^{3}+5 x\right) \mu_{5}-\left(32 x^{6}-48 x^{4}+18 x^{2}-1\right) \mu_{6}>0
\end{aligned}
$$

respectively, for all $x \in[-1,1]$. In Table 2.1, we list several multipliers satisfying these conditions.

Furthermore, evaluating $P$ at $x=3 / 40$, we have

$$
P(3 / 40)<-15.1563+13.7341 \sum_{i=1}^{6}\left|\mu_{i}\right| .
$$

Assuming $\left|\mu_{1}\right|+\cdots+\left|\mu_{6}\right| \leqslant 1$, we observe that

$$
P(3 / 40)<-1.4222<0,
$$

and infer that no Nevanlinna-Odeh multiplier exists for the six-step BDF method. 
TABLE 2.1. Multipliers for the six-step BDF method; see also (2.3).

\begin{tabular}{|c|c|c|c|c|c|}
\hline$\mu_{1}$ & $\mu_{2}$ & $\mu_{3}$ & $\mu_{4}$ & $\mu_{5}$ & $\mu_{6}$ \\
\hline \hline 1.6 & -0.92 & 0.3 & 0 & 0 & 0 \\
\hline 0.8235 & -0.855 & 0.38 & 0 & 0 & 0 \\
\hline 1.67 & -1 & 0.4 & -0.1 & 0 & 0 \\
\hline 0.8 & -0.7 & 0.2 & 0.1 & 0 & 0 \\
\hline 1.118 & -1 & 0.6 & -0.2 & 0.2 & 0 \\
\hline 0.6708 & -0.2 & -0.2 & 0.6 & -0.2 & 0 \\
\hline 0.735 & -0.2 & -0.4 & 0.8 & -0.4 & 0.2 \\
\hline
\end{tabular}

\section{StabiLity}

In this section we prove stability of the six-step BDF method (1.3) by the energy technique. The result is well known; the novelty is in the simplicity of the proof, the main advantage of the energy technique. Proofs by other stability techniques are significantly more involved. For a proof by a spectral technique in the case of selfadjoint operators, we refer to [13, chapter 10]; for a proof in the general case, under a sharp condition on the nonselfadjointness of the operator as well as for nonlinear parabolic equations, by a combination of spectral and Fourier techniques, see, e.g., [2] and references therein. For a long-time estimate in the case of selfadjoint operators and an application to the StokesDarcy problem, see [10].

For simplicity, we denote by $\langle\cdot, \cdot\rangle$ the inner product on $V,\langle v, w\rangle:=\left(A^{1 / 2} v, A^{1 / 2} w\right)$.

Before we proceed, for the reader's convenience, we recall the notion of the generating function of an $n \times n$ Toeplitz matrix $T_{n}$ as well as an auxiliary result, the Grenander-Szegö theorem.

Definition 3.1 ([7, p. 13]; the generating function of a Toeplitz matrix). Consider the $n \times n$ Toeplitz matrix $T_{n}=\left(t_{i j}\right) \in \mathbb{C}^{n, n}$ with diagonal entries $t_{0}$, subdiagonal entries $t_{1}$, superdiagonal entries $t_{-1}$, and so on, and $(n, 1)$ and $(1, n)$ entries $t_{n-1}$ and $t_{1-n}$, respectively, i.e., the entries $t_{i j}=t_{i-j}, i, j=1, \ldots, n$, are constant along the diagonals of $T_{n}$. Let $t_{-n+1}, \ldots, t_{n-1}$ be the Fourier coefficients of the trigonometric polynomial $f$, i.e.,

$$
t_{k}=\frac{1}{2 \pi} \int_{-\pi}^{\pi} f(x) \mathrm{e}^{-\mathrm{i} k x} \mathrm{~d} x, \quad k=1-n, \ldots, n-1 .
$$

Then, $f, f(x)=\sum_{k=1-n}^{n-1} t_{k} \mathrm{e}^{\mathrm{i} k x}$, is called generating function of $T_{n}$.

If the generating function $f$ is real-valued, then the matrix $T_{n}$ is Hermitian; if $f$ is real-valued and even, then $T_{n}$ is symmetric.

Notice, in particular, that the generating function of a symmetric band Toeplitz matrix of bandwidth $2 m+1$, i.e., with $t_{m+1}=\cdots=t_{n-1}=0$, is a real-valued, even trigonometric polynomial, $f(x)=t_{0}+2 t_{1} \cos x+\cdots+2 t_{m} \cos (m x)$, for all $n \geqslant m+1$.

Lemma 3.1 ([7, pp. 13-14]; the Grenander-Szegö theorem). Let $T_{n}$ be a symmetric Toeplitz matrix as in Definition 3.1 with generating function $f$. Then, the smallest and largest eigenvalues $\lambda_{\min }\left(T_{n}\right)$ and $\lambda_{\max }\left(T_{n}\right)$, respectively, of $T_{n}$ are bounded as follows

$$
f_{\min } \leqslant \lambda_{\min }\left(T_{n}\right) \leqslant \lambda_{\max }\left(T_{n}\right) \leqslant f_{\max },
$$


with $f_{\min }$ and $f_{\max }$ the minimum and maximum of $f$, respectively. In particular, if $f_{\min }$ is positive, then the symmetric matrix $T_{n}$ is positive definite. ${ }^{1}$

Theorem 3.1 (Stability of the six-step BDF method). The six-step BDF method (1.3) is stable in the sense that

$$
\left|u^{n}\right|^{2}+\tau \sum_{\ell=6}^{n}\left\|u^{\ell}\right\|^{2} \leqslant C \sum_{j=0}^{5}\left(\left|u^{j}\right|^{2}+\tau\left\|u^{j}\right\|^{2}\right), \quad n=6, \ldots, N,
$$

with a constant $C$ independent of $\tau$ and $n$.

Proof. Taking in (1.3) the inner product with $u^{n+6}-\frac{13}{9} u^{n+5}+\frac{25}{36} u^{n+4}-\frac{1}{9} u^{n+3}$, cf. (2.1), we have

$$
\left(\sum_{i=0}^{6} \alpha_{i} u^{n+i}, u^{n+6}-\sum_{j=1}^{3} \mu_{j} u^{n+6-j}\right)+\tau I_{n+6}=0
$$

with

$$
I_{n+6}:=\left\langle u^{n+6}, u^{n+6}-\sum_{j=1}^{3} \mu_{j} u^{n+6-j}\right\rangle .
$$

With the notation $\mathcal{U}^{n}:=\left(u^{n-5}, u^{n-4}, u^{n-3}, u^{n-2}, u^{n-1}, u^{n}\right)^{\top}$ and the norm $\left|\mathcal{U}^{n}\right|_{G}$ given by

$$
\left|\mathcal{U}^{n}\right|_{G}^{2}=\sum_{i, j=1}^{6} g_{i j}\left(u^{n-6+i}, u^{n-6+j}\right)
$$

using $(\mathrm{G})$, we have

$$
\left(\sum_{i=0}^{6} \alpha_{i} u^{n+i}, u^{n+6}-\sum_{j=1}^{3} \mu_{j} u^{n+6-j}\right) \geqslant\left|\mathcal{U}^{n+6}\right|_{G}^{2}-\left|\mathcal{U}^{n+5}\right|_{G}^{2} .
$$

Thus, (3.2) yields

$$
\left|\mathcal{U}^{n+6}\right|_{G}^{2}-\left|\mathcal{U}^{n+5}\right|_{G}^{2}+\tau I_{n+6} \leqslant 0 .
$$

Summing in (3.5) from $n=0$ to $n=m-6$, we obtain

$$
\left|\mathcal{U}^{m}\right|_{G}^{2}-\left|\mathcal{U}^{5}\right|_{G}^{2}+\tau \sum_{n=6}^{m} I_{n} \leqslant 0 .
$$

It remains to estimate the sum $\sum_{n=6}^{m} I_{n}$ from below; we have

$$
\sum_{n=6}^{m} I_{n}=\sum_{n=6}^{m}\left\langle u^{n}, u^{n}-\sum_{j=1}^{3} \mu_{j} u^{n-j}\right\rangle \text {. }
$$

${ }^{1}$ For real-valued $f$ and $z=\left(z_{0}, \ldots, z_{n-1}\right)^{\top} \in \mathbb{C}^{n}$, we have $\left(T_{n} z, z\right)=\frac{1}{2 \pi} \int_{-\pi}^{\pi} f(x)\left|\sum_{k=0}^{n-1} z_{k} \mathrm{e}^{\mathrm{i} k x}\right|^{2} \mathrm{~d} x$ and $(z, z)=\frac{1}{2 \pi} \int_{-\pi}^{\pi}\left|\sum_{k=0}^{n-1} z_{k} \mathrm{e}^{\mathrm{i} k x}\right|^{2} \mathrm{~d} x$, and the result is evident. 
First, motivated by the positivity of the function $f$ of (2.11), to take advantage of the positivity property (P2), we introduce the notation $\mu_{0}:=-31 / 32$, and rewrite (3.7) as

$$
\sum_{n=6}^{m} I_{n}=\frac{1}{32} \sum_{n=6}^{m}\left\|u^{n}\right\|^{2}+J_{m} \quad \text { with } \quad J_{m}:=-\sum_{j=0}^{3} \mu_{j} \sum_{i=1}^{m-5}\left\langle u^{5+i}, u^{5+i-j}\right\rangle .
$$

Our next task is to rewrite $J_{m}$ in a form that will enable us to estimate it from bellow in a desired way. To this end, we introduce the lower triangular Toeplitz matrix $L=\left(\ell_{i j}\right) \in$ $\mathbb{R}^{m-5, m-5}$ with entries

$$
\ell_{i, i-j}=-\mu_{j}, \quad j=0,1,2,3, \quad i=j+1, \ldots, m-5,
$$

and all other entries equal zero. With this notation, we have

$$
\sum_{i, j=1}^{m-5} \ell_{i j}\left\langle u^{5+i}, u^{5+j}\right\rangle=-\sum_{j=0}^{3} \mu_{j} \sum_{i=j+1}^{m-5}\left\langle u^{5+i}, u^{5+i-j}\right\rangle,
$$

i.e.,

$$
\sum_{i, j=1}^{m-5} \ell_{i j}\left\langle u^{5+i}, u^{5+j}\right\rangle=J_{m}+\left\langle u^{6}, \mu_{1} u^{5}+\mu_{2} u^{4}+\mu_{3} u^{3}\right\rangle+\left\langle u^{7}, \mu_{2} u^{5}+\mu_{3} u^{4}\right\rangle+\left\langle u^{8}, \mu_{3} u^{5}\right\rangle
$$

Now, in view of the positivity of the generating function $f$, see (2.11), of the symmetric part $L_{s}:=\left(L+L^{\top}\right) / 2$ of the matrix $L$, the Grenander-Szegö theorem, see Lemma 3.1, ensures positive definiteness of $L_{s}$, and thus also of $L$ itself, since $(L x, x)=\left(L_{s} x, x\right)$ for $x \in \mathbb{R}^{m-5}$. Therefore, the expression on the left-hand side of (3.9) is nonnegative; hence, (3.9) yields the desired estimate for $J_{m}$ from below, i.e.,

$$
J_{m} \geqslant-\left\langle u^{6}, \mu_{1} u^{5}+\mu_{2} u^{4}+\mu_{3} u^{3}\right\rangle-\left\langle u^{7}, \mu_{2} u^{5}+\mu_{3} u^{4}\right\rangle-\left\langle u^{8}, \mu_{3} u^{5}\right\rangle
$$

From (3.6), (3.8) and (3.10), we obtain

$$
\begin{aligned}
\left|\mathcal{U}^{m}\right|_{G}^{2}+\frac{1}{32} \sum_{n=6}^{m}\left\|u^{n}\right\|^{2} & \leqslant\left|\mathcal{U}^{5}\right|_{G}^{2}+\tau\left\langle u^{6}, \mu_{1} u^{5}+\mu_{2} u^{4}+\mu_{3} u^{3}\right\rangle \\
& +\tau\left\langle u^{7}, \mu_{2} u^{5}+\mu_{3} u^{4}\right\rangle+\tau\left\langle u^{8}, \mu_{3} u^{5}\right\rangle .
\end{aligned}
$$

Now, with $c_{1}$ and $c_{2}$ the smallest and largest eigenvalues of the matrix $G$, we have

$$
\left|\mathcal{U}^{m}\right|_{G}^{2} \geqslant c_{1}\left|u^{m}\right|^{2} \quad \text { and } \quad\left|\mathcal{U}^{5}\right|_{G}^{2} \leqslant c_{2} \sum_{j=0}^{5}\left|u^{j}\right|^{2}
$$

furthermore, the terms $\left|\left\langle u^{i}, u^{j}\right\rangle\right|$ with $i>j$ can be estimated in the form $\left|\left\langle u^{i}, u^{j}\right\rangle\right| \leqslant$ $\varepsilon\left\|u^{i}\right\|^{2}+\left\|u^{j}\right\|^{2} /(4 \varepsilon)$ with $\varepsilon<1 / 32$. This leads then to the desired stability estimate (3.1).

Let us also note that, due to the fact that $\mu_{4}=\mu_{5}=\mu_{6}=0$, the terms $\left\|u^{2}\right\|^{2},\left\|u^{1}\right\|^{2}$ and $\left\|u^{0}\right\|^{2}$ are actually not needed on the right-hand side of (3.1).

3.1. Time-dependent operators. In this section we use a perturbation argument to extend the stability result to the case of time-dependent selfadjoint operators $A(t): V \rightarrow$ $V^{\prime}, t \in[0, T]$. We fix an $s \in[0, T]$ and define the norm on $V$ in terms of $A(s),\|v\|:=$ $\left|A(s)^{1 / 2} v\right|$. 
Our structural assumptions are that all operators $A(t), t \in[0, T]$, share the same domain, produce equivalent norms on $V$,

$$
\left|A(t)^{1 / 2} v\right| \leqslant c\left|A(\tilde{t})^{1 / 2} v\right| \quad \forall t, \tilde{t} \in[0, T] \forall v \in V,
$$

and $A(t): V \rightarrow V^{\prime}$ is of bounded variation with respect to $t$,

$$
\|(A(t)-A(\tilde{t})) v\|_{\star} \leqslant[\sigma(t)-\sigma(\tilde{t})]\|v\|, \quad 0 \leqslant \tilde{t} \leqslant t \leqslant T, \quad \forall v \in V,
$$

with an increasing function $\sigma:[0, T] \rightarrow \mathbb{R}$.

First, for given perturbation terms $v^{6}, \ldots, v^{N} \in V^{\prime}$, we let $u^{6}, \ldots, u^{N}$ satisfy the perturbed six-step BDF method

$$
\sum_{i=0}^{6} \alpha_{i} u^{n+i}+\tau A u^{n+6}=\tau v^{n+6}, \quad n=0, \ldots, N-6,
$$

i.e., the scheme (1.3) for $q=6$ with perturbed right-hand side, assuming that starting approximations $u^{0}, \ldots, u^{5}$ are given. Then, it is easily seen that we have the following stability result

$$
\left|u^{n}\right|^{2}+\tau \sum_{\ell=6}^{n}\left\|u^{\ell}\right\|^{2} \leqslant C \sum_{j=0}^{5}\left(\left|u^{j}\right|^{2}+\tau\left\|u^{j}\right\|^{2}\right)+C \tau \sum_{\ell=6}^{n}\left\|v^{\ell}\right\|_{\star}^{2}, \quad n=6, \ldots, N,
$$

with a constant $C$ independent of $\tau$ and $n$. Indeed, the terms that are due to the perturbation $v^{n+6}$, namely, $\left(v^{n+6}, u^{n+6}-\sum_{j=1}^{3} \mu_{j} u^{n+6-j}\right)$, can be easily estimated in the form

$$
\left(v^{n+6}, u^{n+6}-\sum_{j=1}^{3} \mu_{j} u^{n+6-j}\right) \leqslant C_{\varepsilon}\left\|v^{n+6}\right\|_{\star}^{2}+\varepsilon \sum_{j=1}^{3}\left|\mu_{j}\right|\left\|u^{n+6-j}\right\|^{2},
$$

with sufficiently small $\varepsilon$ such that the terms involving $\left\|u^{i}\right\|^{2}$ can be absorbed in the corresponding sum on the left-hand side.

We shall next use (3.14) to extend the stability result (3.1) to the case of time-dependent operators. Before that, let us note that with $v^{\ell}=f\left(t^{\ell}\right),(3.14)$ is a stability result for the inhomogeneous equation $u^{\prime}(t)+A u(t)=f(t)$ with respect to both the starting approximations and the forcing term. Furthermore, with $v^{\ell}$ the consistency error of the method, i.e., the amount by which the exact solution $u$ misses satisfying the numerical method (1.3), with $q=6$,

$$
\tau v^{n+6}=\sum_{i=0}^{6} \alpha_{i} u\left(t^{n+i}\right)+\tau A u\left(t^{n+6}\right)-\tau f\left(t^{n+6}\right), \quad n=0, \ldots, N-6,
$$

the error $e^{\ell}:=u\left(t^{\ell}\right)-u^{\ell}, \ell=0, \ldots, N$, satisfies (3.13). In this case, the stability result (3.14), in combination with the trivial estimate of the consistency error, leads to optimal order error estimates.

Now, the six-step BDF method for the initial value problem (1.1) with time-dependent operator $A(t)$ is

$$
\sum_{i=0}^{6} \alpha_{i} u^{n+i}+\tau A\left(t^{n+6}\right) u^{n+6}=0, \quad n=0, \ldots, N-6
$$


assuming that starting approximations $u^{0}, \ldots, u^{5}$ are given. Let us now fix an $6 \leqslant m \leqslant N$. From (3.16), we obtain

$$
\sum_{i=0}^{6} \alpha_{i} u^{n+i}+\tau A\left(t^{m}\right) u^{n+6}=\tau\left[A\left(t^{m}\right)-A\left(t^{n+6}\right)\right] u^{n+6}, \quad n=0, \ldots, m-6 .
$$

Since the time $t$ is frozen at $t^{m}$ in the operator $A\left(t^{m}\right)$ on the left-hand side, we can apply the already-established stability estimate (3.14) with perturbation terms $v^{\ell}:=\left[A\left(t^{m}\right)-A\left(t^{\ell}\right)\right] u^{\ell}$ and obtain

$$
\left|u^{m}\right|^{2}+\tau \sum_{\ell=6}^{m}\left\|u^{\ell}\right\|^{2} \leqslant C \sum_{j=0}^{5}\left(\left|u^{j}\right|^{2}+\tau\left\|u^{j}\right\|^{2}\right)+C M^{m}
$$

with a constant $C$ independent of $\tau$ and $m$, and

$$
M^{m}:=\tau \sum_{\ell=6}^{m}\left\|\left[A\left(t^{m}\right)-A\left(t^{\ell}\right)\right] u^{\ell}\right\|_{\star}^{2} .
$$

Now, with

$$
E^{\ell}:=\tau \sum_{j=6}^{\ell}\left\|u^{j}\right\|^{2}, \quad \ell=6, \ldots, m, \quad E^{5}:=0,
$$

estimate (3.18) yields

$$
E^{m} \leqslant C \sum_{j=0}^{5}\left(\left|u^{j}\right|^{2}+\tau\left\|u^{j}\right\|^{2}\right)+C M^{m}
$$

Furthermore, in view of the bounded variation condition (3.12),

$$
M^{m} \leqslant \tau \sum_{\ell=6}^{m-1}\left[\sigma\left(t^{m}\right)-\sigma\left(t^{\ell}\right)\right]^{2}\left\|u^{\ell}\right\|^{2}=\sum_{\ell=6}^{m-1}\left[\sigma\left(t^{m}\right)-\sigma\left(t^{\ell}\right)\right]^{2}\left(E^{\ell}-E^{\ell-1}\right),
$$

whence, by summation be parts, we have

$$
M^{m} \leqslant \sum_{\ell=6}^{m-1} a_{\ell} E^{\ell},
$$

with $a_{\ell}:=\left[\sigma\left(t^{m}\right)-\sigma\left(t^{\ell}\right)\right]^{2}-\left[\sigma\left(t^{m}\right)-\sigma\left(t^{\ell+1}\right)\right]^{2}$, and (3.20) yields

$$
E^{m} \leqslant C \sum_{j=0}^{5}\left(\left|u^{j}\right|^{2}+\tau\left\|u^{j}\right\|^{2}\right)+C \sum_{\ell=6}^{m-1} a_{\ell} E^{\ell} .
$$

Since the sum $\sum_{\ell=6}^{m-1} a_{\ell}$ is uniformly bounded by a constant independent of $m$ and the time step $\tau$,

$$
\sum_{\ell=6}^{m-1} a_{\ell}=\left[\sigma\left(t^{m}\right)-\sigma\left(t^{6}\right)\right]^{2} \leqslant[\sigma(T)-\sigma(0)]^{2},
$$

a discrete Gronwall-type argument applied to (3.22) leads to

$$
E^{m} \leqslant C \sum_{j=0}^{5}\left(\left|u^{j}\right|^{2}+\tau\left\|u^{j}\right\|^{2}\right) .
$$


Combining (3.18) with (3.21) and (3.23), we obtain the desired stability estimate (3.1) for the case of time-dependent operators.

\section{REFERENCES}

1. G. Akrivis, Stability of implicit-explicit backward difference formulas for nonlinear parabolic equations, SIAM J. Numer. Anal. 53 (2015) 464-484.

2. G. Akrivis, Stability of implicit and implicit-explicit multistep methods for nonlinear parabolic equations, IMA J. Numer. Anal. 38 (2018) 1768-1796.

3. G. Akrivis and E. Katsoprinakis, Backward difference formulae: New multipliers and stability properties for parabolic equations, Math. Comp. 85 (2016) 2195-2216.

4. G. Akrivis and E. Katsoprinakis, Maximum angles of $A(\vartheta)$-stability of backward difference formulae, BIT Numer. Math. 60 (2020) 93-99.

5. G. Akrivis and C. Lubich, Fully implicit, linearly implicit and implicit-explicit backward difference formulae for quasi-linear parabolic equations, Numer. Math. 131 (2015) 713-735.

6. C. Baiocchi and M. Crouzeix, On the equivalence of A-stability and G-stability, Appl. Numer. Math. 5 (1989) 19-22.

7. R. H. Chan and X. Q. Jin, An Introduction to Iterative Toeplitz Solvers, SIAM, Philadelphia, PA, 2007.

8. G. Dahlquist, G-stability is equivalent to A-stability, BIT 18 (1978) 384-401.

9. E. Hairer and G. Wanner, Solving Ordinary Differential Equations II: Stiff and Differential-Algebraic Problems, $2^{\text {nd }}$ revised ed., Springer-Verlag, Berlin Heidelberg, Springer Series in Computational Mathematics v. 14, 2010.

10. B. Li, K. Wang, and Z. Zhou, Long-time accurate symmetrized implicit-explicit BDF methods for a class of parabolic equations with non-self-adjoint operators, SIAM J. Numer. Anal. 58 (2020) 189-210.

11. C. Lubich, D. Mansour, and C. Venkataraman, Backward difference time discretization of parabolic differential equations on evolving surfaces, IMA J. Numer. Anal. 33 (2013) 1365-1385.

12. O. Nevanlinna and F. Odeh, Multiplier techniques for linear multistep methods, Numer. Funct. Anal. Optim. 3 (1981) 377-423.

13. V. Thomée, Galerkin Finite Element Methods for Parabolic Problems, $2^{\text {nd }}$ ed., Springer-Verlag, Berlin, 2006.

Department of Computer Science and Engineering, University of IoAnnina, 45110 IoAnnina, Greece, and Institute of Applied and Computational Mathematics, FORTH, 70013 Heraklion, Crete, Greece

E-mail address: akrivis@cse.uoi.gr

School of Mathematics and Statistics, Gansu Key Laboratory of Applied Mathematics and Complex Systems, Lanzhou University, Lanzhou 730000, P.R. China

E-mail address: chenmh@lzu.edu.cn

School of Mathematics and Statistics, Gansu Key Laboratory of Applied Mathematics and Complex Systems, Lanzhou University, Lanzhou 730000, P.R. China

E-mail address: yuf17@1zu.edu.cn

Department of Applied Mathematics, The Hong Kong Polytechnic University, Kowloon, Hong Kong, P.R. China

E-mail address: zhizhou@polyu.edu.hk 